\title{
Heat conduction in a composite sphere - the effect of fractional derivative order on temperature distribution
}

\author{
Urszula Siedlecka ${ }^{1, *}$, Stanisław Kukla ${ }^{1}$ \\ ${ }^{1}$ Institute of Mathematics, Czestochowa University of Technology, Częstochowa, Poland
}

\begin{abstract}
The aim of the contribution is an analysis of time-fractional heat conduction in a sphere with an inner heat source. The object of the consideration is a solid sphere with a spherical layer. The heat conduction in the solid sphere and spherical layer is governed by fractional heat conduction equation with a Caputo timederivative. Mathematical (classical) or physical formulations of the Robin boundary condition and the perfect contact of the solid sphere and spherical layer is assumed. The boundary condition and the heat flux continuity condition at the interface are expressed by the Riemann-Liouville derivative. An exact solution of the problem under mathematical conditions is determined. A solution of the problem under physical boundary and continuity conditions using the Laplace transform method has been obtained. The inverse of the Laplace transform by using the Talbot method are numerically determined. Numerical results show the effect of the order of the Caputo and the Riemann-Liouville derivatives on the temperature distribution in the sphere.
\end{abstract}

Keywords: fractional heat conduction, heating source, Robin boundary condition

\section{Introduction}

The fundamental of the classical heat transfer theory is the Fourier law which leads to the parabolic partial differential equation of the heat conduction [1]. A consequence of the Fourier's law is unrealistic speed of heat flow in the medium. This inconvenience can be avoided by a generalization of the Fourier law which leads to a fractional heat conduction equation [2]. The heat conduction governed by the fractional differential equation is the subject of papers [3-9]. Applications of fractional order calculus are presented in books [10-12] and papers [13-15].

If the heat transfer in a bounded medium is considered then the heat equation is complemented by boundary conditions. The Dirichlet, Neumann and Robin boundary conditions are often used in describing the heat transfer between the body and the surroundings. In the classical heat theory, the Neumann and Robin boundary conditions include the normal derivative at the boundary of the considered region. Introducing the time-fractional derivative in the Neumann and Robin boundary conditions, the physical formulation of these conditions is obtained [16].

\footnotetext{
* Corresponding author: urszula.siedlecka@im.pcz.pl

Reviewers: Ján Vavro Jr., Branislav Ftorek
} 
A solution of the linear fractional differential equation under classical boundary conditions can be determined in an analytical form. To solve the fractional equation under physical boundary conditions, the Laplace technique can be applied. This approach leads to a solution of the problem in the Laplace domain. The temperature distribution in the time domain by using an algorithm for numerical inversion of the Laplace transform can be obtained. The methods of numerical inversion of the Laplace transforms used in classical analysis can be also applied to Laplace transforms obtained by solving the problems with fractional derivatives. Selected methods of numerical inversion of the Laplace transforms are presented in papers [17-20].

In this paper, we present the solution of the fractional heat conduction problem in a sphere consisting of an inner solid sphere and a spherical layer. The mathematical and physical formulation of the Robin boundary conditions is considered. The perfect thermal contact of the inner sphere and the spherical layer is assumed. The effect of the fractional order on the temperature distribution in the sphere has been numerically investigated.

\section{Formulation of the problem}

We consider the time-fractional radial heat conduction problem in a sphere. The two regions of the sphere are distinguished: $0 \leq r \leq r_{1}$ - a solid inner sphere and $r_{1} \leq r \leq b$ - a spherical layer, where $r$ is the radial coordinate. The heat transfer in the regions is governed by the fractional heat conduction equation [3]:

$$
\frac{1}{r^{2}} \frac{\partial}{\partial r}\left(r^{2} \frac{\partial T_{i}}{\partial r}\right)+\frac{1}{\lambda_{i}} g_{i}(r, t)=\frac{1}{a_{i}} \frac{\partial^{\alpha_{i}} T_{i}}{\partial t^{\alpha_{i}}}, \quad i=1,2
$$

where $g_{i}(r, t)$ is the volumetric rate of heat generation, $a_{i}$ is the thermal diffusivity, $\lambda_{i}$ is the thermal conductivity and $\alpha_{i}$ denotes the fractional order of the Caputo derivative with respect to time $t$. The Caputo derivative is defined by [21]

$$
{ }_{0}^{C} D_{t}^{\alpha} f(t)=\frac{d^{\alpha}}{d t^{\alpha}} f(t)= \begin{cases}\frac{1}{\Gamma(m-\alpha)} \int_{0}^{t} \frac{f^{(m)}(\tau)}{(t-\tau)^{\alpha+1-m}} d \tau, & m-1<\alpha \leq m \\ f^{(m)}(t), & \alpha=m \in N\end{cases}
$$

where $\Gamma$ denotes the gamma function. The boundary condition and the continuity condition at interface are assumed in a form with the Riemann-Liouville fractional derivative $D_{R L}^{1-\beta}$ which is defined by [21]

$$
D_{R L}^{\beta} f(t)=\frac{d}{d t}\left[\frac{1}{\Gamma(1-\beta)} \int_{0}^{t} \frac{f(\tau)}{(t-\tau)^{\beta}} d \tau\right], \quad 0<\beta \leq 1
$$

On the outer surface of the sphere, the Robin boundary condition [16] is assumed

$$
\lambda_{2} D_{R L}^{1-\beta_{2}} \frac{\partial T_{2}}{\partial r}(b, t)=a_{\infty}\left(T_{\infty}(t)-T_{2}(b, t)\right)
$$


where $a_{\infty}$ is the outer heat transfer coefficient and $T_{\infty}$ is the ambient temperature. The perfect thermal contact at the interface between the inner sphere and the spherical layer is described by conditions:

$$
\begin{gathered}
T_{1}\left(r_{1}, t\right)=T_{2}\left(r_{1}, t\right) \\
\lambda_{1} D_{R L}^{1-\beta_{1}} \frac{\partial T_{1}}{\partial r}\left(r_{1}, t\right)=\lambda_{2} D_{R L}^{1-\beta_{2}} \frac{\partial T_{2}}{\partial r}\left(r_{1}, t\right)
\end{gathered}
$$

and the initial condition is

$$
T(r, 0)=F_{i}(r)
$$

The conditions (4) and (6) for $\beta_{1}=\alpha_{1}$ and $\beta_{2}=\alpha_{2}$ are called the physical conditions [16], if $\beta_{1}=\beta_{2}=1$, there are so called mathematical conditions. In the second case, the $D_{R L}^{0}$ in equations (4) and (6) means an identity operator and can be omitted. We further consider the case of $\alpha_{i} \in(0,1]$.

\section{Solution to the problem}

In order to transform the heat conduction equation (1) into the fractional equation with a constant coefficient, we introduce new functions $U_{i}(r, t)$ given by the following relationship

$$
U_{i}(r, t)=r\left(T_{i}(r, t)-T_{\infty}(t)\right), \quad i=1,2
$$

Taking into account equation (8) in the initial-boundary problem (1) and (4-7), we obtain formulation of the problem for the function $U_{i}(r, t)$ in the form

$$
\begin{gathered}
a_{i} \frac{\partial^{2} U_{i}(r, t)}{\partial r^{2}}+r g_{i}^{*}(r, t)=\frac{\partial^{\alpha_{i}} U_{i}(r, t)}{\partial t^{\alpha_{i}}}, \quad i=1,2 \\
\lambda_{2} D_{R L}^{1-\beta_{2}}\left(\frac{\partial U_{2}}{\partial r}(b, t)-\frac{1}{b} U_{2}(b, t)\right)=-a_{\infty} U_{2}(b, t) \\
U_{1}\left(r_{1}, t\right)=U_{2}\left(r_{1}, t\right) \\
\lambda_{1} D_{R L}^{1-\beta_{1}}\left(\frac{\partial U_{1}\left(r_{1}, t\right)}{\partial r}-\frac{1}{r_{1}} U_{1}\left(r_{1}, t\right)\right)=\lambda_{2} D_{R L}^{1-\beta_{2}}\left(\frac{\partial U_{2}\left(r_{1}, t\right)}{\partial r}-\frac{1}{r_{1}} U_{2}\left(r_{1}, t\right)\right) \\
U_{i}(r, 0)=r\left(F_{i}(r)-T_{\infty}(0)\right), \quad i=1,2
\end{gathered}
$$

Moreover, the conditions (10-12) are complemented by a condition for $r=0$, which is obtained using equation (8) 


$$
U_{1}(0, t)=0
$$

The functions $g_{i}^{*}(r, t)$ in equation (9) are given by the formula

$$
g_{i}^{*}(r, t)=\frac{a_{i}}{\lambda_{i}} g_{i}(r, t)-\frac{d^{\alpha_{i}} T_{\infty}(t)}{d t^{\alpha_{i}}}
$$

The solutions of the initial-boundary problem (9-14) for mathematical and physical formulations will be presented below.

\subsection{Mathematical formulation of boundary and continuity conditions}

The heat conduction problem (9-14) under mathematical conditions for $\alpha_{1}=\alpha_{2}=\alpha$ can be solved analytically. We search for the solution to this problem in the form of the series of orthogonal functions $\Psi_{i, k}(r)$ :

$$
U_{i}(r, t)=\sum_{k=1}^{\infty} \Lambda_{k}(t) \Psi_{i, k}(r), \quad i=1,2
$$

In the first step, we find the functions $\Psi_{i, k}(r)$ by solving the following eigenvalue problem

$$
\begin{gathered}
\frac{d^{2} \Psi_{i, k}(r)}{d r^{2}}+\frac{\gamma_{k}^{2}}{a_{i}} \Psi_{i, k}(r)=0 \quad i=1,2 \\
\Psi_{1, k}(0)=0 \\
\Psi_{1, k}\left(r_{1}\right)=\Psi_{2, k}\left(r_{1}\right) \\
\lambda_{1}\left(\frac{d \Psi_{1}\left(r_{1}\right)}{d r}-\frac{1}{r_{1}} \Psi_{1}\left(r_{1}\right)\right)=\lambda_{2}\left(\frac{d \Psi_{2}\left(r_{1}\right)}{d r}-\frac{1}{r_{1}} \Psi_{2}\left(r_{1}\right)\right) \\
\frac{d \Psi_{2}(b)}{d r}=\left(\frac{1}{b}-\frac{a_{\infty}}{\lambda_{2}}\right) \Psi_{2}(b)
\end{gathered}
$$

The functions $\Psi_{i, k}(r)$ are

$$
\begin{gathered}
\Psi_{1, k}(r)=B_{1, k} \sin \mu_{1, k} r \\
\Psi_{2, k}(r)=A_{2, k} \cos \mu_{2, k}\left(r-r_{1}\right)+B_{2, k} \sin \mu_{2, k}\left(r-r_{1}\right)
\end{gathered}
$$

where $\mu_{i, k}=\gamma_{k} / \sqrt{a_{i}}$ and $\gamma_{k}$ are roots of the equation

$$
Q_{1} \lambda_{2} \mu_{1} \sin \mu_{1} r_{1}+Q_{2} Q_{3}=0
$$

where 


$$
\begin{aligned}
& Q_{1}=\left(\frac{a_{\infty}}{\lambda_{2}}-\frac{1}{b}\right) \cos \mu_{2}\left(b-r_{1}\right)-\mu_{2} \sin \mu_{2}\left(b-r_{1}\right), \\
& Q_{2}=\left(\frac{a_{\infty}}{\lambda_{2}}-\frac{1}{b}\right) \sin \mu_{2}\left(b-r_{1}\right)+\mu_{2} \cos \mu_{2}\left(b-r_{1}\right)
\end{aligned}
$$

and

$$
Q_{3}=\lambda_{1} \mu_{1} \cos \mu_{1} r_{1}+\frac{\lambda_{2}-\lambda_{1}}{r_{1}} \sin \mu_{1} r_{1}
$$

These functions fulfil the orthogonality condition in the form

$$
\frac{\lambda_{1}}{a_{1}} \int_{0}^{r_{1}} \Psi_{1, k}(r) \Psi_{1, k^{\prime}}(r) d r+\frac{\lambda_{2}}{a_{2}} \int_{r_{1}}^{b} \Psi_{2, k}(r) \Psi_{2, k^{\prime}}(r) d r= \begin{cases}0 & \text { for } k^{\prime} \neq k \\ N_{k} & \text { for } k^{\prime}=k\end{cases}
$$

The coefficients $B_{1, k}, A_{2, k}, B_{2, k}$, occurring in equations (22-23), are determined by using conditions (19-21). Assuming $B_{1, k}=1$, we obtain $A_{2, k}=\sin \mu_{1, k} r_{1} \quad$ and $B_{2, k}=Q_{3} / \lambda_{2} \mu_{2, k}$.

The function $\Lambda_{k}(t)$ satisfies a fractional equation which is obtained substituting equation (16) into equation (9) and using orthogonality condition (25). The initial condition for the function $\Lambda_{k}(t)$ is obtained in a similar way by using (16) and (25) in condition (13). The fractional differential equation and initial condition have the form

$$
\begin{gathered}
\frac{d^{\alpha} \Lambda_{k}(t)}{d t^{\alpha}}+\gamma_{k}^{2} \Lambda_{k}(t)=\frac{1}{N_{k}^{r}}\left(\int_{0}^{r_{1}} r g_{1}^{*}(r, t) \Psi_{1, k}(r) d r+\int_{r_{1}}^{b} r g_{2}^{*}(r, t) \Psi_{2, k}(r) d r\right) \\
\Lambda_{k}(0)=\frac{1}{N_{k}^{r}}\left(\frac{\lambda_{1}}{a_{1}} \int_{0}^{r_{1}} r\left(F_{1}(r)-T_{\infty}(0)\right) \Psi_{1, k}(r) d r+\frac{\lambda_{2}}{a_{2}} \int_{r_{1}}^{b} r\left(F_{2}(r)-T_{\infty}(0)\right) \Psi_{2, k}(r) d r\right)
\end{gathered}
$$

where $N_{k}^{r}=\frac{\lambda_{1}}{a_{1}} \int_{0}^{r_{1}}\left[\Psi_{1, k}(r)\right]^{2} d r+\frac{\lambda_{2}}{a_{2}} \int_{r_{1}}^{b}\left[\Psi_{2, k}(r)\right]^{2} d r$. A solution of the problem (26-27) is given by [22]

$$
\begin{aligned}
& \Lambda_{k}(t)=\frac{1}{N_{k}^{r}}\left(\int_{0}^{r_{1}} r \Psi_{1, k}(r) \int_{0}^{t}(t-\tau)^{\alpha-1} g_{1}^{*}(r, \tau) E_{\alpha, \alpha}\left(-\gamma_{k}^{2}(t-\tau)^{\alpha}\right) d \tau d r\right. \\
& \left.\quad+\int_{r_{1}}^{b} r \Psi_{2, k}(r) \int_{0}^{t}(t-\tau)^{\alpha-1} g_{2}^{*}(r, \tau) E_{\alpha, \alpha}\left(-\gamma_{k}^{2}(t-\tau)^{\alpha}\right) d \tau d r\right) \\
& +\frac{1}{N_{k}^{r}} E_{\alpha, 1}\left(-\gamma_{k}^{2} t^{\alpha}\right)\left(\frac{\lambda_{1}}{a_{1}} \int_{0}^{r_{1}} r\left(F_{1}(r)-T_{\infty}(0)\right) \Psi_{1, k}(r) d r+\frac{\lambda_{2}}{a_{2}} \int_{r_{1}}^{b} r\left(F_{2}(r)-T_{\infty}(0)\right) \Psi_{2, k}(r) d r\right)
\end{aligned}
$$

where $E_{\alpha}(z)$ is the Mittag-Leffler function defined by 


$$
E_{\alpha}(z)=\sum_{k=0}^{\infty} \frac{z^{k}}{\Gamma(\alpha k+1)}
$$

Finally, taking into account equation (8) and (16), we obtain the temperature distribution in the sphere under mathematical formulation of the boundary and continuity conditions in the form

$$
T_{i}(r, t)=T_{\infty}(t)+\frac{1}{r} \sum_{k=1}^{\infty} \Lambda_{k}(t) \Psi_{i, k}(r), \quad i=1,2
$$

where functions $\Lambda_{k}(t)$ are given by (28) and $\Psi_{i, k}(r)$ are defined by (22) and (23). If $F_{i}(r)=T_{\text {init }}=$ const,$T_{\infty}(t)=T_{\infty}=$ const and $g_{1}(r, t)=G=$ const,$g_{2}(r, t)=0$, then the functions $\Lambda_{k}(t)$ are given by

$$
\begin{aligned}
& \Lambda_{k}(t)=\frac{G}{N_{k}^{r} \mu_{1, k}^{2} \gamma_{k}^{2}} \frac{a_{1}}{\lambda_{1}}\left(\sin \mu_{1, k} r_{1}-\mu_{1, k} r_{1} \cos \mu_{1, k} r_{1}\right)\left(1-E_{\alpha}\left(-\gamma_{k}^{2} t^{\alpha}\right)\right) \\
& \quad+\frac{T_{\text {init }}-T_{\infty}}{N_{k}^{r}} E_{\alpha}\left(-\gamma_{k}^{2} t^{\alpha}\right)\left\{\frac{\lambda_{1}}{\mu_{1, k}^{2} a_{1}}\left(\sin \mu_{1, k} r_{1}-\mu_{1, k} r_{1} \cos \mu_{1, k} r_{1}\right)+\frac{\lambda_{2}}{a_{2}} \frac{1}{\mu_{2, k}^{2}} .\right. \\
& \quad\left[A_{2, k}\left(b \mu_{2, k} \sin \mu_{2, k}\left(b-r_{1}\right)+\cos \mu_{2, k}\left(b-r_{1}\right)-1\right)\right. \\
& \left.\left.\quad+B_{2, k}\left(-b \mu_{2, k} \cos \mu_{2, k}\left(b-r_{1}\right)+\sin \mu_{2, k}\left(b-r_{1}\right)+r_{1} \mu_{2, k}\right)\right]\right\}
\end{aligned}
$$

\subsection{Physical formulation of boundary and continuity conditions}

A solution of the heat conduction problem (9-14) under physical boundary and continuity conditions ( $\beta_{1}=\alpha_{1}, \beta_{2}=\alpha_{2}$ in equations (10) and (12)) will be obtained by using the Laplace technique. The Laplace transform is defined as

$$
\bar{f}(s)=\int_{0}^{\infty} f(t) e^{-s t} d t
$$

where $f(t)$ for $t>0$ is a given function of the exponential type and $s$ is a complex parameter. After applying the Laplace transformation to the equations (9-12) and (14), and using the properties of the Laplace transform, we obtain

$$
\begin{gathered}
\frac{d^{2} \bar{U}_{i}}{d r^{2}}-\frac{s^{\alpha_{i}}}{a_{i}} \bar{U}_{i}=\bar{h}_{i}(r, s) \\
\bar{U}_{1}(0, s)=0 \\
\bar{U}_{1}\left(r_{1}, s\right)=\bar{U}_{2}\left(r_{1}, s\right) \\
\lambda_{1} s^{1-\beta_{1}}\left(\frac{d \bar{U}_{1}\left(r_{1}, s\right)}{d r}-\frac{1}{r_{1}} \bar{U}_{1}\left(r_{1}, s\right)\right)=\lambda_{2} s^{1-\beta_{2}}\left(\frac{d \bar{U}_{2}\left(r_{1}, s\right)}{d r}-\frac{1}{r_{1}} \bar{U}_{2}\left(r_{1}, s\right)\right)
\end{gathered}
$$




$$
\frac{d \bar{U}_{2}(b, s)}{d r}+\left(\frac{a_{\infty}}{\lambda_{2} s^{1-\beta_{2}}}-\frac{1}{b}\right) \bar{U}_{2}(b, s)=0
$$

where

$$
\bar{h}_{i}(r, s)=r \frac{s^{\alpha_{i}-1}}{a_{i}}\left(s \bar{T}_{\infty}(s)-F_{i}(r)\right)-\frac{r}{\lambda_{i}} \bar{g}_{i}(r, s) .
$$

The solution of the equation (33) for $i=1$, using the condition (34), can be written in the form

$$
\bar{U}_{1}(r, s)=B_{1} \sinh S_{1} r+\frac{1}{S_{1}} \int_{0}^{r} \bar{h}_{1}(u, s) \sinh S_{1}(r-u) d u
$$

and the general solution of equation (33) for $i=2$, is as follows

$$
\bar{U}_{2}(r, s)=A_{2} \cosh S_{2}\left(r-r_{1}\right)+B_{2} \sinh S_{2}\left(r-r_{1}\right)+\frac{1}{S_{2}} \int_{r_{1}}^{r} \bar{h}_{2}(u, s) \sinh S_{2}(r-u) d u
$$

where $S_{i}=\frac{s^{\alpha_{i} / 2}}{\sqrt{a_{i}}}$. Using conditions (35-37), a system of linear equations with respect to unknown constants $B_{1}, A_{2}$ and $B_{2}$ is obtained

$$
\begin{gathered}
B_{1} \sinh S_{1} r_{1}-A_{2}=P_{1} \\
B_{1} s^{1-\beta_{1}} \lambda_{1}\left(S_{1} \cosh S_{1} r_{1}-\frac{1}{r_{1}} \sinh S_{1} r_{1}\right)+A_{2} \frac{s^{1-\beta_{2}} \lambda_{2}}{r_{1}}-B_{2} s^{1-\beta_{2}} \lambda_{2} S_{2}=P_{2} \\
A_{2} \frac{1}{s^{\beta_{2}}}\left(a_{\infty} b s^{\beta_{2}} S_{2} \cosh S_{2}\left(b-r_{1}\right)-s S_{2} \lambda_{2} \cosh S_{2}\left(b-r_{1}\right)+b s S_{2}^{2} \lambda_{2} \sinh S_{2}\left(b-r_{1}\right)\right) \\
+B_{2} \frac{1}{s^{\beta_{2}}}\left(b s S_{2}^{2} \lambda_{2} \cosh S_{2}\left(b-r_{1}\right)+a_{\infty} b s^{\beta_{2}} S_{2} \sinh S_{2}\left(b-r_{1}\right)-s S_{2} \lambda_{2} \sinh S_{2}\left(b-r_{1}\right)\right)=P_{3} \\
\text { where } \\
P_{1}=-\frac{1}{S_{1}} \int_{0}^{r_{1}} \overline{h_{1}}(u, s) \sinh S_{1}\left(r_{1}-u\right) d u \\
P_{2}=-\frac{s^{1-\beta_{1}} \lambda_{1}}{S_{1} r_{1}}\left(S_{1} r_{1} \int_{0}^{r_{1}} \overline{h_{1}}(u, s) \cosh S_{1}\left(r_{1}-u\right) d u-\int_{0}^{r_{1}} \overline{h_{1}}(u, s) \sinh S_{1}\left(r_{1}-u\right) d u\right), \\
s^{\beta_{2}}\left(s \lambda_{2} S_{2} b \int_{r_{1}}^{b} \bar{h}_{2}(u, s) \cosh S_{2}(b-u) d u+\left(a_{\infty} b s^{\beta_{2}}-s \lambda_{2}\right) \int_{r_{1}}^{b} \bar{h}_{2}(u, s) \sinh S_{2}(b-u) d u\right) .
\end{gathered}
$$

Substituting the determined constants $B_{1}, A_{2}$ and $B_{2}$ into equations (38-39), we get the complete solution of the problem in the Laplace domain. The temperature distribution in the sphere is given by 


$$
T_{i}(r, t)=T_{\infty}(t)+\frac{1}{r} L^{-1}\left[\bar{U}_{i}(r, s)\right], \quad i=1,2
$$

The inverse of the Laplace transform of the functions $\bar{U}_{i}(r, s), i=1,2$ is calculated numerically. The fixed Talbot algorithm to numerical inversion of the Laplace transform has been used, [18-19]. Applying this algorithm, the approximate values of the function $U_{i}(r, t)=L^{-1}\left[\bar{U}_{i}(r, s)\right]$ are determined using the formula

$$
U_{i}(r, t)=\frac{p}{M}\left\{\frac{1}{2} \bar{U}_{i}(r, p) \exp (p t)+\sum_{k=1}^{M-1} \operatorname{Re}\left[\exp \left(t s\left(\theta_{k}\right)\right) \bar{U}_{i}\left(r, s\left(\theta_{k}\right)\right)\left(1+j \sigma\left(\theta_{k}\right)\right)\right]\right\}
$$

where $s(\theta)=p \theta(\operatorname{ctg} \theta+j), \quad \sigma(\theta)=\theta+(\theta \operatorname{ctg} \theta-1) \operatorname{ctg} \theta, \quad p=2 M /(5 t), \quad j=\sqrt{-1}$, and $\theta_{k}=k \pi / M, M$ is a number of precision decimal digits.

\section{Results of numerical calculations}

The effect of the fractional order of time-derivatives occurring in the heat conduction equation and boundary conditions on the temperature distribution in the sphere has been numerically investigated. For the heat conduction model with the mathematical formulation of the boundary and continuity conditions, the results obtained by using the numerical inversion of the Laplace transform has been compared with the exact solution. The calculation has been performed for the following geometrical and thermal data: outer radius of the sphere is $b=1.0[\mathrm{~m}]$, the radius of the inner sphere is $\hat{r}_{1}=r_{1} / b=0.6$, the thermal diffusivities are $a_{1}=3.352 \cdot 10^{-6}\left[\mathrm{~m}^{2} / \mathrm{s}^{\alpha}\right], \quad a_{2}=1.474 \cdot 10^{-5}\left[\mathrm{~m}^{2} / \mathrm{s}^{\alpha}\right]$, the thermal conductivities are $\lambda_{1}=16[\mathrm{~W} /(\mathrm{m} \cdot \mathrm{K})], \lambda_{2}=54[\mathrm{~W} /(\mathrm{m} \cdot \mathrm{K})]$, the outer heat transfer coefficient is $a_{\infty}=500\left[\mathrm{~W} /\left(\mathrm{m}^{2} \cdot \mathrm{K}\right)\right]$, the ambient temperature is $T_{\infty}=25\left[{ }^{\circ} \mathrm{C}\right]$ and the initial temperature is assumed as $T_{\text {init }}=25\left[{ }^{\circ} \mathrm{C}\right]$.

Table 1. The non-dimensional temperature $\hat{T}(\hat{r}, \hat{t})$ for $\hat{t}=t b^{2} / a_{2}=1.0$, calculated by using the exact solution and numerical inversion of the Laplace transform (NILT)

\begin{tabular}{|c|c|c|c|c|c|c|c|c|}
\hline \multirow{2}{*}{$\hat{r}$} & \multicolumn{2}{|c|}{$\alpha=0.7$} & \multicolumn{2}{c|}{$\alpha=0.8$} & \multicolumn{2}{c|}{$\alpha=0.9$} & \multicolumn{2}{c|}{$\alpha=1.0$} \\
\cline { 2 - 9 } & Exact & NILT & Exact & NILT & Exact & NILT & Exact & NILT \\
\hline 0 & 1.44421 & 1.44404 & 2.25696 & 2.25679 & 3.73501 & 3.73484 & 4.77135 & 4.77118 \\
\hline 0.2 & 1.44176 & 1.44175 & 2.20899 & 2.20898 & 3.53158 & 3.53158 & 4.44462 & 4.44462 \\
\hline 0.4 & 1.40995 & 1.40995 & 1.98699 & 1.98699 & 2.87100 & 2.87100 & 3.46198 & 3.46198 \\
\hline 0.6 & 1.13143 & 1.13143 & 1.32024 & 1.32023 & 1.61769 & 1.61768 & 1.81594 & 1.81594 \\
\hline 0.8 & 1.03183 & 1.03182 & 1.11964 & 1.11962 & 1.27291 & 1.27289 & 1.37645 & 1.37643 \\
\hline 1.0 & 1.00653 & 1.00652 & 1.03246 & 1.03245 & 1.08066 & 1.08065 & 1.11348 & 1.11347 \\
\hline
\end{tabular}


The non-dimensional temperature $\hat{T}=T / T_{\text {init }}$ in the selected points of the sphere for the mathematical boundary and continuity conditions $\left(\beta_{1}=\beta_{2}=1.0, \alpha_{1}=\alpha_{2}=\alpha\right)$ for different orders of the Caputo derivative $\alpha$ are presented in Table 1. The volumetric rate of heat generation in the inner sphere is $g_{1}=20000\left[\mathrm{~W} / \mathrm{m}^{3}\right]$ and in the spherical layer $g_{2}=0$. The results presented in the Table 1 fulfil the condition: $\mid$ Exact $-N I L T \mid /$ Exact $<3.6 \cdot 10^{-5}$. The small differences of the Exact and NILT results allow for the use of the algorithm of numerical inversion of the Laplace transform to the heat conduction problem under physical formulation of boundary and continuity conditions.
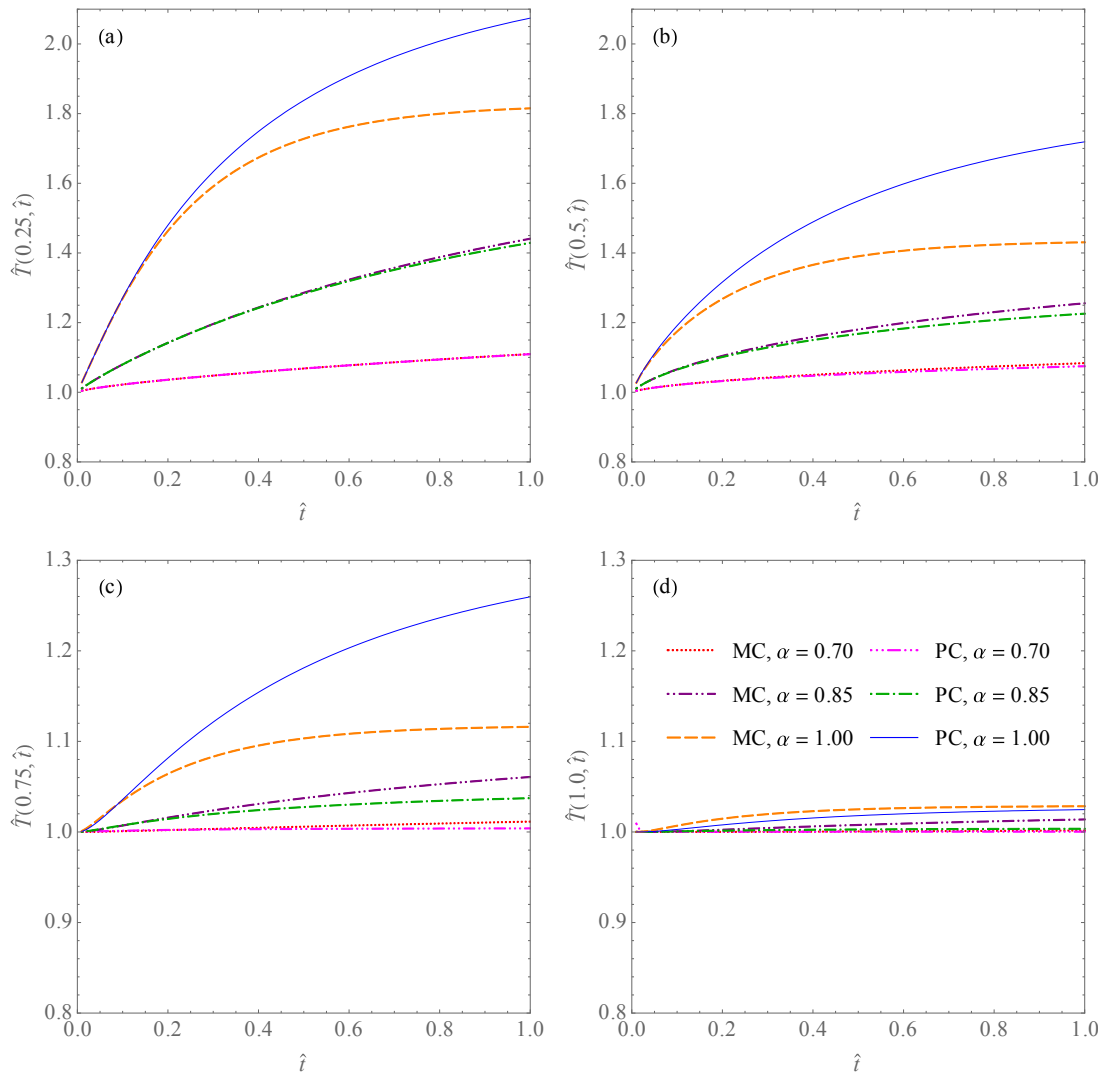

Fig. 1. The non-dimensional temperature $\hat{T}(\hat{r}, \hat{t})$ as a function of time $\hat{t}$ for various values of fractional derivatives $\alpha$ and $\beta$ : (a) $\hat{r}=0.25$; (b) $\hat{r}=0.5$; (c) $\hat{r}=0.75$; (d) $\hat{r}=1.0$

The time-histories temperatures in selected points of the sphere are presented in Fig. 1. The calculations were performed for different orders of the derivative occurring in the heat equation. In the problem under mathematical conditions (MC) one was assumed $\alpha_{1}=\alpha_{2}=\alpha=0.7 ; 0.85 ; 1.0$ and $\beta_{1}=\beta_{2}=1.0$ and for the problem under physical conditions (PC) the calculations were performed for $\alpha_{1}=\alpha=0.7 ; 0.85 ; 1.0, \alpha_{2}=\beta_{2}=0.9$ and $\beta_{1}=\alpha_{1}$. The volumetric rate of heat generation was assumed : $g_{1}=5000\left[\mathrm{~W} / \mathrm{m}^{3}\right]$ and $g_{2}=0$. As was expected, the differences between the temperatures obtained for various orders of $\alpha$ 's decrease if the distance from the source increases. The significant effect on 
the temperature distribution in the sphere has order of the derivatives in the heat conduction model.

\section{Conclusions}

The problem of the fractional heat conduction in a sphere with inner heat source under mathematical and physical Robin boundary and continuity conditions by using the Laplace transform technique has been solved. It was noted a high agreement of the results obtained on the basis of the exact solution and the results computed by using numerical inversion of the Laplace transform for the heat conduction under mathematical conditions. The effect of the order of the Caputo derivative occurring in the heat conduction equation on the temperature distribution in the sphere has been numerically investigated. It was stated that the temperatures in the sphere obtained for models with the same fractional order $\alpha$ with mathematical and physical Robin conditions differ slightly. The significant differences in the temperatures have been observed for different orders of the fractional derivative in the heat conduction equation.

\section{References}

1. M.N. Özişik, Heat conduction. (Wiley, New York, 1993)

2. Y. Povstenko, Fractional heat conduction in a semi-infinite composite body. Communications in Applied and Industrial Mathematics 6 (1), e-482 (2014)

3. Y. Povstenko, Fractional heat conduction in an infinite medium with a spherical inclusion. Entropy 15, 4122-4133 (2013)

4. Y. Povstenko, J. Klekot, The fundamental solutions to the central symmetric timefractional heat conduction equation with heat absorption. Journal of Applied Mathematics and Computational Mechanics 16 (2), 101-112 (2017)

5. S. Blasiak, Time-fractional heat transfer equations in modeling of the non-contacting face seals. International Journal of Heat and Mass Transfer 100, 79-88 (2016)

6. T.H. Ning, X.Y. Jiang, Analytical solution for the time-fractional heat conduction equation in spherical coordinate system by the method of variable separation. Acta Mechanica Sinica 27 (6), 994-1000 (2011)

7. S. Kukla, U. Siedlecka, Laplace transform solution of the problem of time-fractional heat conduction in a two-layered slab. Journal of Applied Mathematics and Computational Mechanics 14 (4), 105-113 (2015)

8. S. Kukla, U. Siedlecka, An analytical solution to the problem of time-fractional heat conduction in a composite sphere. Bulletin of the Polish Academy of Sciences Technical Sciences 65 (2), 179-186 (2017)

9. U. Siedlecka, Radial heat conduction in a multilayered sphere. Journal of Applied Mathematics and Computational Mechanics 13 (4), 109-116 (2014)

10. T. M. Atanacković, S. Pilipović, B. Stanković, D. Zorica, Fractional Calculus with Applications in Mechanics. (John Wiley \& Sons, New York, 2014)

11. A. A. Kilbas, H. M. Srivastava, J. J. Trujillo, Theory and applications of fractional differential equations. (Elsevier, Amsterdam, 2006)

12. J.S. Leszczyński, An Introduction to Fractional Mechanics. The Publishing Office of Czestochowa University of Technology, Czestochowa (2011) 
13. M. Dalir, M. Bashour, Applications of fractional calculus. Applied Mathematical Sciences 4 (21), 1021-1032 (2010)

14. A. Dzieliński, D. Sierociuk, G. Sarwas, Some applications of fractional order calculus. Bulletin of the Polish Academy of Sciences - Technical Sciences 58 (4), 583-592 (2010)

15. W. E. Raslan, Application of fractional order theory of thermoelasticity to a $1 D$ problem for a spherical shell. Journal of Theoretical and Applied Mechanics 54 (1), 295-304 (2016)

16. Y. Povstenko, Time-fractional heat conduction in an infinite medium with a spherical hole under Robin boundary condition. Fractional Calculus \& Applied Analysis 16 (2), 354-369 (2013)

17. K. L. Kuhlman, Review of inverse Laplace transform algorithms for Laplace-space numerical approaches. Numerical Algorithms 63 (2), 339-355 (2013)

18. J. Abate, P. P. Valkó, Multi-precision Laplace transform inversion. International Journal for Numerical Methods in Engineering 60, 979-993 (2004)

19. B. Dingfelder, J. A. C. Weideman, An improved Talbot method for numerical Laplace transform inversion. Numerical Algorithms 68, 167-183 (2015)

20. H. Sheng, Y. Li, Y. Chen, Application of numerical inverse Laplace transform algorithms in fractional calculus. Journal of the Franklin Institute 384, 315-330 (2011)

21. I. Podlubny, Fractional differential equations. (Academic Press, San Diego, 1999)

22. K. Diethelm, The analysis of fractional differential equations. (Springer-Verlag Berlin Heidelberg, 2010) 